\title{
Continuous ultrasonic thickness measurement technology
}

\author{
Vitaliy Pronin $^{1}$, Petr Shkatov ${ }^{1}$, Anna Sandulyak ${ }^{1, *}$, and Haci Mehmet Baskonus ${ }^{2}$ \\ ${ }^{1}$ Moscow technological university, Moscow, RF \\ ${ }^{2}$ Department of Computer Engineering, Munzur University, Tunceli, Turkey
}

\begin{abstract}
The article describes the technologies and means of ultrasonic thickness measurement for pipeline base metal and welded joints using phased array antennas with linear scanning by TOFD and DAF methods. According to the thickness measurement data provided through specialized software, maps are obtained of the control objects thickness. The measurement results are given using these technologies, as well as the prospects for these methods development.
\end{abstract}

\section{Introduction}

Pipelines in operation are subject to erosion-corrosion wear. Under the influence of the heat carrier, erosion of the protective oxide film destruction occurs on the inner surface, which contributes to the corrosion process, occurrence of corrosion cracks resulting in metal destruction of the pipeline wall.

To ensure reliable and safe pipelines operation, it is necessary to use modern achievements in ultrasonic nondestructive testing and diagnostics of pipeline walls metal and welded joints. One of these directions is continuous ultrasonic thickness measurement of the base metal and welded seams using antenna arrays (AA). Unlike selective ultrasonic thickness measurement, the use of automated scanning devices (SDs) allows identifying and capturing a complete representation of metal condition.

The following technologies and means of ultrasonic thickness measurement have been developed:

- Continuous ultrasonic thickness measurement of the pipelines base metal using antenna arrays.

- Ultrasonic thickness measurement of welded joints according to TOFD (Time-of-flight diffraction) scheme using antenna arrays.

- Ultrasonic thickness measurement of welded joints using digital antenna focusing method with the bottom surface profile plotting.

\section{Continuous ultrasonic thickness measurement of the pipelines base metal}

The control object (QA) is the base metal of monometallic pipelines, bends and conical transitions. The range of controlled pipeline wall thicknesses is 5.5 mm.

\footnotetext{
Corresponding author: anna.sandulyak@mail.ru
}

The relatively wide measurement strip of the pipeline thickness measurement is ensured by a control scheme where AA moves along the axis of the pipeline and oriented across the direction of scanning (Fig. 1). AA are used in the process of measurement, installed on flatbent delays with the contact concave surface (Fig. 2). To carry out the thickness measurement of the pipelines base metal, a mechanized two-coordinate scanning device (SD) was developed on magnetic wheels according to the proposed control scheme (Fig. 3).

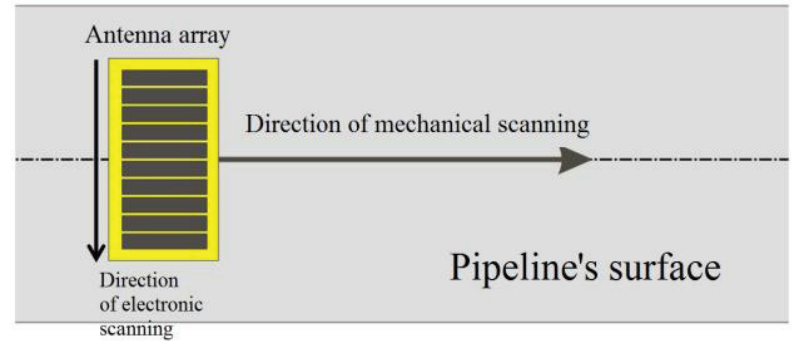

Fig. 1. Thickness measurement scheme for pipelines base metal.

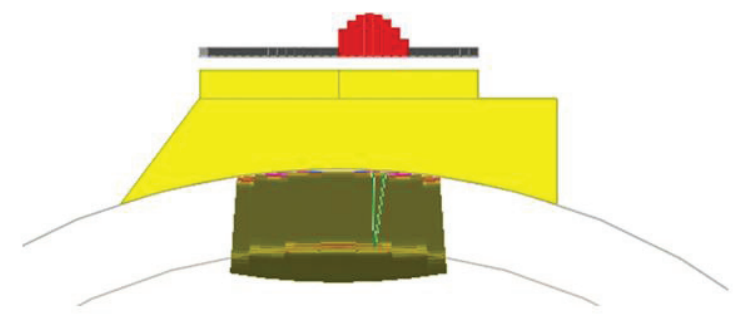

Fig. 2. Electronic scanning along AA aperture.

Specialized software (SW) was developed to handle the thickness measurement data. SW interface is shown in Fig. 4.

An example of measurements and resulting thickness map is shown in Fig. 5. 


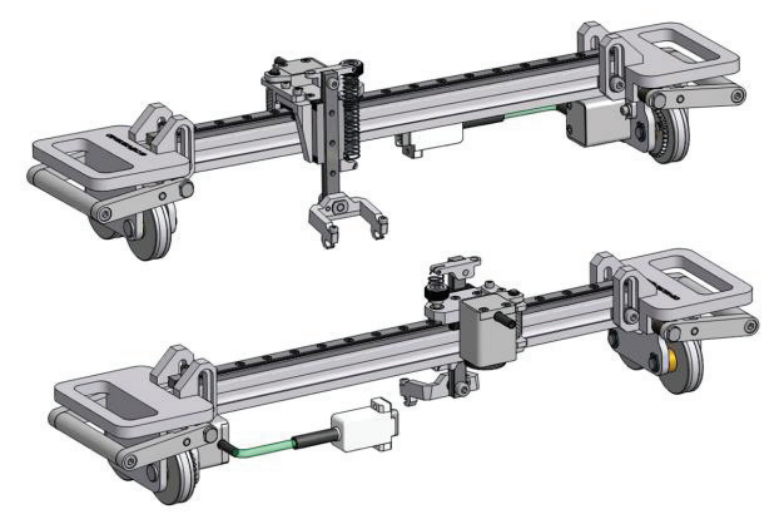

Fig. 3. Scanner for the base metal thickness measurement.

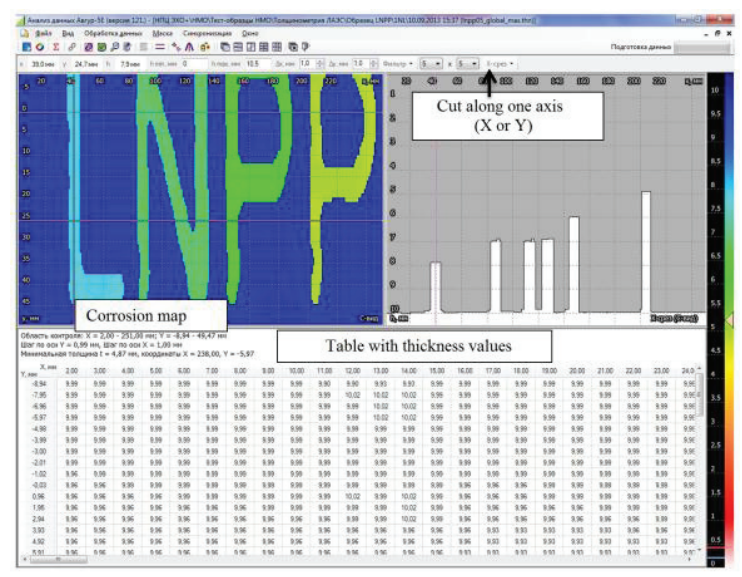

Fig. 4. Software interface for building and analyzing thickness maps.

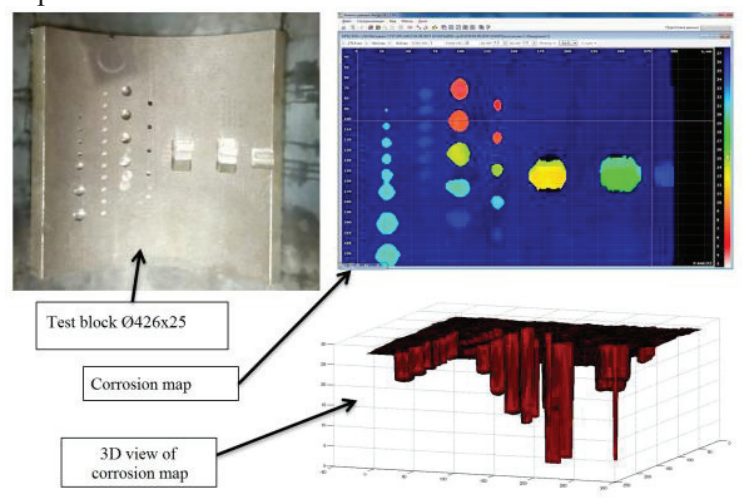

Fig. 5. Test specimen $\varnothing 426 \times 25 \mathrm{~mm}$ with a thickness map built on the basis of thickness measurements using AA.

\section{Ultrasonic thickness measurement of welded joints according to TOFD (Time- of-flight diffraction) scheme using antenna arrays}

The control objects according to this method are welded joints of pipelines with external diameters from $\varnothing 159 \mathrm{~mm}$ and wall thickness of $5.5 \mathrm{~mm}$.

The measurements are carried out using ultrasonic phased antenna arrays according to the ultrasonic time-of-flight diffraction method - TOFD (Fig. 6). At the same time, thickness measurements in the weld proximity are carried out using the same AAs operating in the electronic scanning mode with an input angle of $0^{\circ}$, based on the arrival time of the bottom signal (Fig. 7). Thus, a wide strip of thickness measurement is provided.

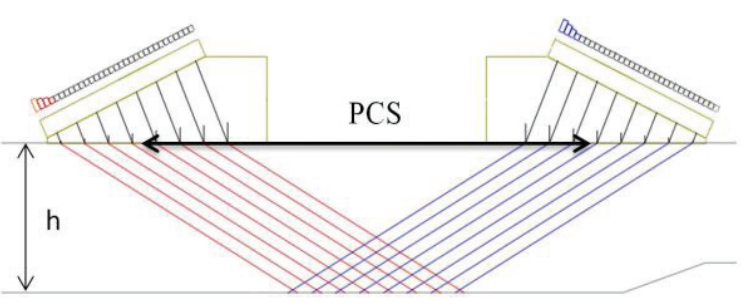

Fig. 6. Principle of thickness measurement in the area of welded joints according to TOFD scheme with AA and electronic scanning.

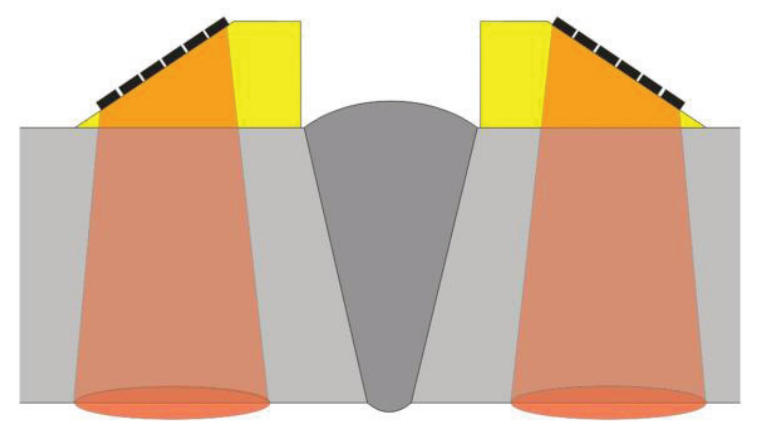

Fig. 7. Principle of thickness measurement in the weld proximity zone.

An unidirectional scanning device with a set of tracks for diameters Ø 159, Ø 219, Ø 273, Ø 325, Ø 377 and Ø 426 mm developed by SRC ECHO+ LLC (Fig. 8) is used to move the antenna arrays.

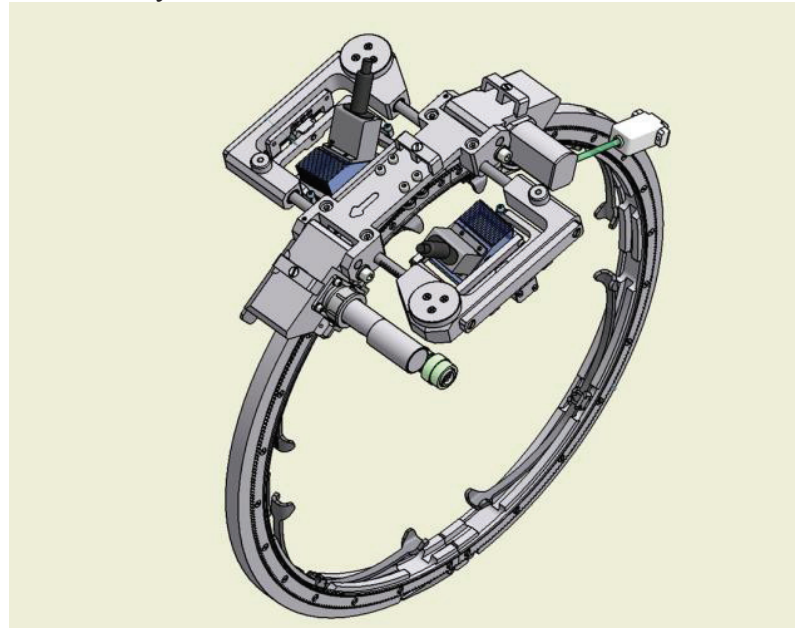

Fig. 8. Scanner SK-TD.160-426.DP-1 with track of Ø $325 \mathrm{~mm}$.

An example of measurements and resulting thickness map is shown in Fig. 9. 


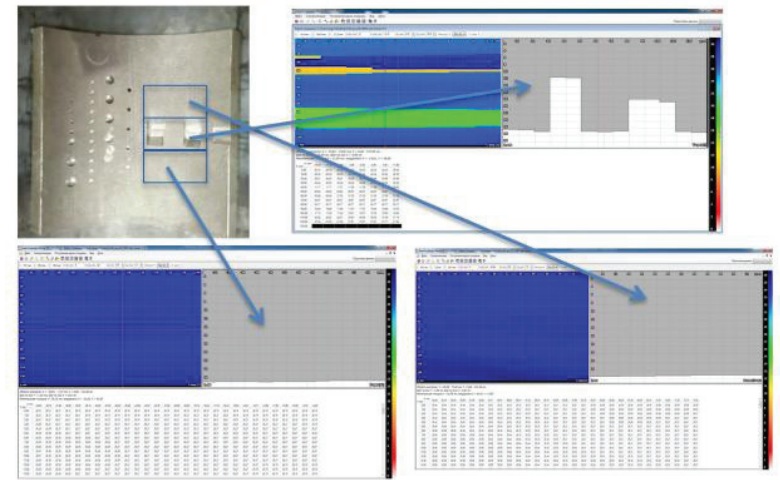

Fig. 9. Test specimen $Ø 426 \times 25 \mathrm{~mm}$ with a thickness map built based on WJ thickness measurements according to TOFD scheme.

\section{Ultrasonic thickness measurement of welded joints using digital antenna focusing method with the bottom surface profile plotting}

To control the thickness of pipelines with non-equidistant surfaces, the [1] technology was developed for the bottom surface profile plotting based on an ultrasonic method with digital antenna focusing (DAF). The method is based on ultrasonic waves emission to $\mathrm{CO}$ and registration of these ultrasonic waves reflected from the internal boundaries of $\mathrm{CO}$, as well as subsequent DAF digital processing [2] with generation of images from the registered signals. The technology is based on the use of two antenna arrays (AA) with two identical prisms according to two schemes: separate scheme and combined scheme (Fig. 10).

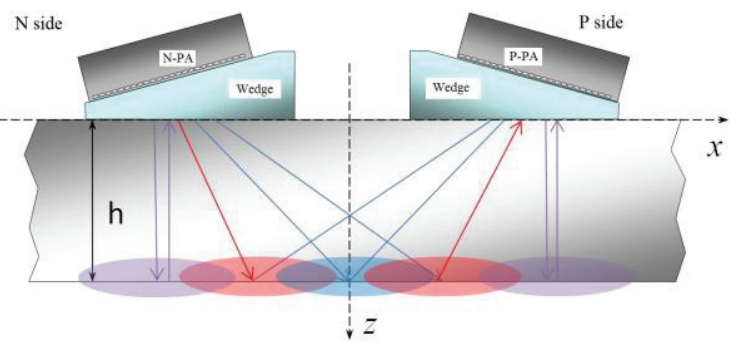

Fig. 10. DAF application for WJ thickness measurement.

Violet color - longitudinal waves according to the combined scheme, red color - longitudinal waves according to the separate scheme, violet color - transverse waves according to the separate scheme.

Then, the received echo signals are processed according to DAF method and total reconstructed image with the bottom surface profile is determined (Fig. 11).

Fig. 12 shows examples of samples thickness measurement with real erosion-corrosive wear (ECW) with built thickness map.

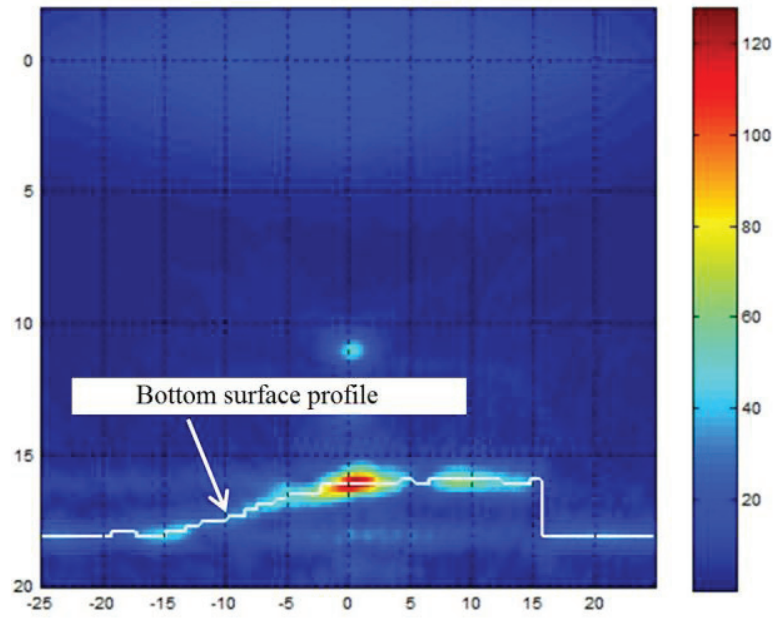

Fig. 11. Total reconstructed image with a bottom surface profile.
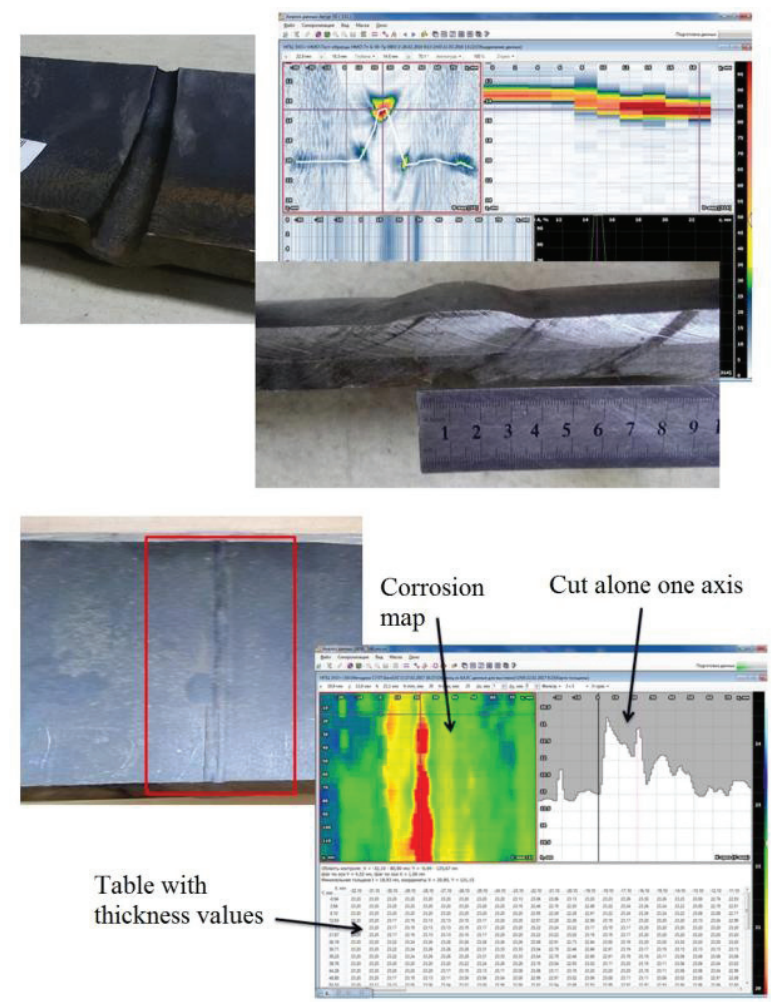

Fig. 12. Application of WJ ultrasonic thickness measurement technology based on DAF samples with ECW.

\section{Conclusion}

- The developed technologies make it possible to detect corrosive wear, edge displacement, thinning, sagging, various thicknesses, etc., in a timely manner when controlling objects with non-equidistant external and internal surfaces, and also to measure the geometrical parameters of the inner surface.

- The proposed technologies can be widely used in ultrasonic flaw detection of pipelines and other metal structures in nuclear power engineering, oil and gas transport infrastructure, chemical engineering, 
shipbuilding with base metal control, as well as with non-equidistant surfaces of the control object.

- Internal surface recording will increase the reliability and efficiency of automated operational control for critical equipment by obtaining high-quality images of reflectors.

The bottom surface profile plotting technology will give impetus to the development of automated methods for recognizing and measuring discontinuities.

\section{Acknowledgements}

The research is conducted with financial support from RFFI within the frameworks of research project № 16-38-60034 mol_a_dk.

\section{References}

1. Way of ultrasonic control of a profile of an internal surface of a product with uneven surfaces. Patent RF № 2560754 (2014).

2. E.G. Basulin, S.A. Kokolev, A.S. Golubev. Defectoskopiya, 7 (2009). 